\title{
NONSMOOTH SET VARIATIONAL INEQUALITY PROBLEMS AND OPTIMALITY CRITERIA FOR SET OPTIMIZATION
}

\author{
E. KARAMAN
}

Received 29 March, 2020

\begin{abstract}
In this work, set-valued optimization problems are considered according to an order relation, which is a partial order on the family such that contains nonempty bounded sets of the space. A generalized convexity is defined for set-valued mapping by using the partial order relation. Nonsmooth variational inequality problems are introduced with the aid of $M$-directionally derivative. Some optimality criteria including the necessary and sufficient optimality conditions are obtained for mentioned optimization problems.
\end{abstract}

2010 Mathematics Subject Classification: 80M50; 90C26; 47J30; 32C22

Keywords: set-valued optimization, variational inequalities, optimality criteria

\section{INTRODUCTION}

One of the most encountered problems in our life is optimization problems (mathematical programming problems). Translating these problems into mathematical language, give us objective functions. Optimization problems are called according to objective functions. For example, a set-valued optimization problem (shortly, $\left(S V O_{P}\right)$ ) arises when the objective function is a set-valued mapping. $\left(S V O_{P}\right)$ s are a generalization of vector and scalar optimization problems because the set-valued mappings are a generalization version of the vector-valued and real-valued functions.

The most important purpose of a mathematical optimization problem is to find the best among the suitable options. Naturally, it has been attracted the attention of scientists, who have been working in mathematics, engineering, economics, management, economic equilibria, optimal control, nonlinear optimization transportation, and many other disciplines. There are many methods to solve and obtain optimality conditions of the optimization problems such as scalarization [14], vectorization [10,12], directional derivative [13], subdifferential [9,11], embedding space [15, 18], variational inequality problems $[1,2,4-8,19,21]$.

Vector variational inequality problems and their some generalizations have been used as methods to obtain the solutions and the optimality conditions of vector-valued optimization problems. Giannessi [8] started the vector variational inequalities. During the most recent decade, different kinds of variational inequality problems were 
derived as Minty variational [2,3,6,7], complementarity [21], semi-monotone scalar variational inequalities [5].

Order relation is required to obtain the solution of set-valued programming and interval programming problems. Set optimization is presented by Kuroiwa [16]. Kuroiwa used six order relation such that some of them are pre-order relation and the others are not pre-order relations. Karaman et al. [14] are defined two order relations. The most important feature of these two order relations is a partial order relation on the family, which contains nonempty bounded sets of the space. These partial order relations are used in [11,13-15] to obtain optimality criteria and solutions for set-valued optimization problems.

The point of this paper is to gain the optimality criteria for $\left(S V O_{P}\right) \mathrm{s}$ via a partial order relation introduced in [14]. Variational inequalities and convexity are used in order to achieve the aim. A new convexity concept called $m$-convexity, which is a generalization of known convexity in the literature, is given by using partial order relation. A relationship is obtained between $m$-convexity and $M$-directional derivative defined in [13].

The layout of this manuscript is as tracks: Some basic notations, definitions, and solution concepts are recalled for $\left(S V O_{P}\right) \mathrm{s}$ in the second section. $m$-convexity and some properties are obtained in Section 3. Variational inequality problems and some optimality conditions including necessary and sufficient are obtained in the last section.

\section{Preliminaries}

Throughout this paper, we assume that $\mathbb{R}^{n}$ is ordered by a convex, pointed, closed cone $C \subseteq \mathbb{R}^{n}(n \geq 1)$ with a nonempty interior. We denote by $\mathcal{P}^{n}$ and $\mathcal{K}^{n}$ the set of all nonempty subsets of $\mathbb{R}^{n}$ and the set of all nonempty compact and convex subsets of $\mathbb{R}^{n}$, respectively. The interior of $A$ is represented by $\operatorname{int}(A)$ for a set $A \subseteq \mathbb{R}^{n}$.

Let $A, B \in \mathcal{P}^{n}$ and $\lambda \in \mathbb{R}, \lambda A:=\{\lambda a \mid a \in A\}$. The algebraic sum and the algebraic difference of $A$ and $B$ are denoted by $A+B$ and $A-B$, respectively. Also, Minkowski (Pontryagin) difference of $A$ and $B$ is defined by

$$
A \dot{-} B:=\left\{x \in \mathbb{R}^{n} \mid x+B \subseteq A\right\} .
$$

The algebraic sum, the algebraic difference and Minkowski difference have following properties.

It is known that $C$ induce the following partial order relation on $\mathbb{R}^{n}$ for $x_{1}, x_{2} \in \mathbb{R}^{n}$ :

$$
x_{1} \leq_{C} x_{2} \Longleftrightarrow x_{2}-x_{1} \in C .
$$

Proposition 1 ([14,17,20]). Let $A, B \in \mathcal{K}^{n}, a \in \mathbb{R}^{n}$ and $t>0$. The following conditions are hold:

(i) $t(A \dot{-} B)=t A \dot{-} t B$

(ii) $(A+B) \dot{-} B=A$, 
(iii) $(A \dot{A} B)+B \subseteq A$,

(iv) if $B=\varnothing$, then $A \dot{-} B=\mathbb{R}^{n}$,

(v) $A \dot{-} A=0$.

Let's now define $m$ and strictly $m$ order relations are recalled in the next definitions.

Definition 1 ([14]). Let $A, B \in \mathcal{P}^{n}$. $m$ order relation is defined by

$$
A \preceq^{m} B: \Longleftrightarrow(B \dot{-} A) \cap C \neq \varnothing .
$$

Note that $\preceq^{m}$ is not only a pre-order relation on $\mathcal{P}^{n}$ but also a partial order relation on $\mathcal{K}^{n}[14]$.

Definition 2 ([14]). Let $A, B \in \mathcal{P}^{n}$. Strictly $m$ order relation is defined by

$$
A \prec^{m} B: \Longleftrightarrow(B \dot{-} A) \cap \operatorname{int}(C) \neq \varnothing .
$$

We know that $m$ and strictly $m$ order relations are not only compatible with the nonnegative scalar multiplication but also compatible with the addition. Moreover, these order relations have the following properties, which are utilized in the next sections.

Proposition 2. Let $A, B, D, E \in \mathcal{P}^{n}$. Then,

(i) $A \preceq^{m} B \Longrightarrow A \dot{-} D \preceq^{m} B \dot{-} D$,

(ii) $A \preceq^{m} B$ and $D \preceq^{m} E \Longrightarrow A+D \preceq^{m} B+E$,

(iii) $A \preceq^{m} B \Longleftrightarrow 0 \preceq^{m} B \dot{-} A \Longrightarrow 0 \preceq^{m} B-A$,

(iv) $A \swarrow^{m} B \Longleftrightarrow \alpha A \swarrow^{m} \alpha B$ for all $\alpha>0$.

Proof. $\quad$ (i) Let $A \preceq^{m} B$. There exists an $s \in C$ that $s \in B \dot{-} A$, it follows

$$
s+A \subseteq B .
$$

Then, $s+A \dot{-} D \subseteq B \dot{-} D$. Really, let $t \in A \dot{-} D$, that is

$$
t+D \subseteq A \text {. }
$$

Then, from (2.1) and (2.2) we have $s+t+D \subseteq s+A \subseteq B$. Hence, $s+t \in B \dot{-} D$ and we obtain $s+A \dot{-} D \subseteq B \dot{-} D$. Since $s \in C$ and $s \in(B \dot{-} D) \dot{-}(A \dot{-} D)$ we obtain $A \dot{-} D \preceq^{m} B \dot{-} D$.

(ii-iv) These can be proved with the aid of Proposition 1 and definitions of $\preceq^{m}$ and $\prec^{m}$.

Note that given all properties via $m$ order relation in Proposition 2 are satisfied for strictly $m$ order relation.

Some efficient sets of a family with aid of $\preceq^{m}$ and $\prec^{m}$ are remembered in the next definition. 
Definition 3 ([14]). Let $\mathcal{S} \subseteq \mathcal{K}^{n}$ and $A \in \mathcal{S}$. Then,

(i) $A$ is called $m$-minimal (resp. $m$-maximal) set of $\mathcal{S}$ if there isn't any $B \in \mathcal{S}$ such that $B \preceq^{m} A$ (resp. $A \preceq^{m} B$ ) and $A \neq B$,

(ii) $A$ is called weakly $m$-minimal (resp. weakly $m$-maximal) set of $\mathcal{S}$ if there isn't any $B \in \mathcal{S}$ such that $B \prec^{m} A$ (resp. $A \prec^{m} B$ ).

Let $S \subseteq \mathbb{R}^{m}$ and $T: S \rightrightarrows \mathbb{R}^{n}$ be a nonempty, compact and convex valued set-valued mapping. Epigraph of the set-valued mapping $T$ is described as $\operatorname{Epi}(T):=\{(x, \alpha) \in$ $\left.S \times \mathbb{R}^{n} \mid T(x) \preceq^{m} \alpha\right\}$. We consider the following constraint $\left(S V O_{P}\right)$

$$
\left(S V O_{P}\right)\left\{\begin{array}{l}
\min (\max ) T(x) \\
\text { s.t. } x \in S .
\end{array}\right.
$$

Set optimization criteria are derived from a comparison between the values of the set-valued mapping $T$. Namely, we search efficient sets of the family $\mathcal{T}(S):=$ $\{T(x) \mid x \in S\}$ to solve a $\left(S V O_{P}\right)$ via set optimization. Ordering relations defined on sets are used to investigate the efficient sets. So, we will use $\preceq^{m}$ and $\prec^{m}$ order relations to determine the efficient sets of $\mathcal{T}(S)$ in this study. If we consider the $\left(S V O_{P}\right)$ with respect to $m$ order relation, then we use the notation $\left(m-S V O_{P}\right)$.

We say that $\hat{x}$ is a minimal (resp. maximal) solution of $\left(m-S V O_{P}\right)$ if $T(\hat{x})$ is an $m$-minimal (resp. $m$-maximal) set of $\mathcal{T}(S)$. Similarly, we say that $\hat{x}$ is a weakly minimal (resp. weakly maximal) solution of $\left(m-S V O_{P}\right)$ if $T(\hat{x})$ is a weakly $m$ minimal (resp. weakly $m$-maximal) set of $\mathcal{T}(S)$. If $T(\hat{x}) \preceq^{m} T(x)$ (resp. $T(x) \preceq^{m}$ $T(\hat{x})$ ) for all $x \in S$, then $\hat{x}$ is called strongly $m$-minimal (resp. strongly $m$-maximal) solution of $\left(m-S V O_{P}\right) . \quad \hat{x}$ is called stritly $m$-minimal (resp. strictly $m$-maximal) solution of $\left(m-S V O_{P}\right)$ if $T(\hat{x}) \prec^{m} T(x)$ (resp. $\left.T(x) \prec^{m} T(\hat{x})\right)$ for all $x \in S$.

If $\hat{x}$ is a strongly $m$-minimal solution of $\left(m-S V O_{P}\right)$, then it is also $m$-minimal and weakly $m$-minimal solution of the problem. Also, if $\hat{x}$ is an $m$-minimal solution of $\left(m-S V O_{P}\right)$, then it is also a weakly $m$-minimal solution of the problem. These conditions can also be applied to maximal solutions.

Definition 4 ([13]). Let $T: \mathbb{R}^{m} \rightrightarrows \mathbb{R}^{n}$ be a set-valued mapping, $S \subseteq \mathbb{R}^{m}, \hat{x} \in \operatorname{int}(S)$ and $h \in \mathbb{R}^{m}$. The limit

$$
T^{M}(\hat{x} ; h):=\limsup _{t \rightarrow 0^{+}} \frac{T(\hat{x}+t h) \dot{-} T(\hat{x})}{t}
$$

is called $M$-directional derivative of $T$ at $\hat{x}$ in direction $h$ where $\limsup _{x^{\prime} \rightarrow x} T\left(x^{\prime}\right):=\{y \in$ $\left.\mathbb{R}^{n} \mid \liminf _{x^{\prime} \rightarrow x} d\left(y, T\left(x^{\prime}\right)\right)=0\right\}$ denotes the Painlevé-Kuratowski upper/outer limit of $T$ at $x$. If $T^{M}(\hat{x} ; h) \neq \varnothing$ for an $\hat{x} \in \operatorname{int}(S)$ and for all $h \in \mathbb{R}^{m}$, then $T$ is called $M$ directionally differentiable at $\hat{x}$.

$T$ is called $M$-directionally differentiable on $S$ if $T^{M}(\hat{x} ; h) \neq \varnothing$ for all $\hat{x} \in \operatorname{int}(S)$ and for all $h \in \mathbb{R}^{m}$. Besides, $T^{M}(\hat{x} ; h)$ is positively homogenous in $h$, that is $T^{M}(\hat{x} ; \alpha h)=$ $\alpha T^{M}(\hat{x} ; h)$ for all $\alpha>0$ [13]. 
Karaman et al. [13] obtained some optimality conditions for $\left(m-S V O_{P}\right)$ and existence theorems for $M$-directional derivative.

\section{3. $M$-CONVEXITY FOR SET-VALUED MAPPINGS}

A convexity notation is introduced for the set-valued mappings by using $m$ order relation, and a relationship between convexity and $M$-directional differentiable is derived in this section.

Definition 5. Let $S \subseteq \mathbb{R}^{m}$ be a convex set and $T: S \rightrightarrows \mathbb{R}^{n}$ be a set-valued mapping. $T$ is called $m$-convex set-valued mapping on $S$ if

$$
T(\lambda x+(1-\lambda) y) \preceq^{m} \lambda T(x)+(1-\lambda) T(y),
$$

for all $x, y \in S$ and all $\lambda \in(0,1)$.

Note that $m$-convexity is reduced the $C$-convexity defined on $\mathbb{R}^{n}$ if we take $f$ : $\mathbb{R}^{m} \rightarrow \mathbb{R}^{n}$ vector-valued function instead of the set-valued mapping $T: \mathbb{R}^{m} \rightrightarrows \mathbb{R}^{n}$. Moreover, if we take $g: \mathbb{R}^{m} \rightarrow \mathbb{R}$ real-valued function instead of the set-valued map $T$, we obtain convexity defined on $\mathbb{R}$. So, $m$-convexity is a generalization of the known convexity in the literature.

Proposition 3. Let $T: \mathbb{R}^{m} \rightrightarrows \mathbb{R}^{n}$ be a set-valued mapping. The following conditions are equivalent:

(i) $T$ is m-convex,

(ii) $\operatorname{Epi}(T)$ is convex,

(iii) $T\left(t_{1} x_{1}+t_{2} x_{2}+\ldots+t_{n} x_{n}\right) \preceq^{m} t_{1} T\left(x_{1}\right)+t_{2} T\left(x_{2}\right)+\ldots+t_{n} T\left(x_{n}\right)$ for all $n \in \mathbb{N}$, for all $x_{1}, x_{2}, \ldots, x_{n} \in \mathbb{R}^{m}$ and for all $t_{1}, t_{2}, \ldots, t_{n} \in(0,1)$ such that $\sum_{k=1}^{n} t_{k}=1$ (Jensen's Inequality).

Proof. The proof is immediate from Definition 5.

Proposition 4. Let $T, G: \mathbb{R}^{m} \rightrightarrows \mathbb{R}^{n}$ be set-valued mappings. The undermentioned assertions are satisfied:

(i) If $T$ is $m$-convex, then $T(x)+C$ and $T(x)+\operatorname{int}(C)$ are $m$-convex,

(ii) if $T$ and $G$ are $m$-convex, $T+G$ is also $m$-convex.

Definition 6. A vector-valued mapping $T: \mathbb{R}^{m} \rightarrow \mathbb{R}^{n}$ is called affine iff

$$
T(\alpha x+(1-\alpha) y)=\alpha T(x)+(1-\alpha) T(y),
$$

for all $x, y \in \mathbb{R}^{m}$ and all $\alpha \in \mathbb{R}$.

Definition 7. Let $h: \mathbb{R}^{m} \rightarrow \mathbb{R}^{n}$ be a vector-valued mapping and $x, y \in \mathbb{R}^{m} . h$ is called

(i) $m$-increasing iff $x \preceq^{m} y$ implies $h(x) \preceq^{m} h(y)$,

(ii) $m$-decreasing iff $x \preceq^{m} y$ implies $h(y) \preceq^{m} h(x)$. 
Proposition 5. Let $T, G: \mathbb{R}^{m} \rightrightarrows \mathbb{R}^{n}$ be set-valued mappings and vector-valued function $h: \mathbb{R}^{k} \rightarrow \mathbb{R}^{m}$ be m-increasing. Then,

(i) If $T$ is $m$-convex set-valued map and $h$ is $m$-convex, then $T \circ h: \mathbb{R}^{k} \rightrightarrows \mathbb{R}^{n}$ is also an m-convex set-valued mapping,

(ii) Let $G$ be an affine set-valued mapping. $T$ is $m$-convex iff $T+G$ is $m$-convex set-valued mapping.

Theorem 1. Let $S \subseteq \mathbb{R}^{m}$ be a convex set and $T: \mathbb{R}^{m} \rightrightarrows \mathbb{R}^{n}$ be compact, convex valued and $M$-directionally differentiable set-valued mapping on $S$. If $T$ is $m$-convex set-valued map, $T^{M}(x ; y-x) \preceq^{m} T(y)-T(x)$ for all $x, y \in S$.

Proof. Because $T$ is $m$-convex set-valued mapping. Then, we have

$$
T(\alpha y+(1-\alpha) x) \preceq^{m} \alpha T(y)+(1-\alpha) T(x)
$$

for all $x, y \in S$ and for all $\alpha \in(0,1)$. As $T$ is convex valued map we can write the inequality (3.1) as

$$
T(x+\alpha(y-x)) \preceq^{m} \alpha T(y)+T(x)-\alpha T(x) .
$$

From Proposition 2 (iii) we get

$$
T(x+\alpha(y-x)) \dot{-} T(x) \preceq^{m}[\alpha T(y)-\alpha T(x)+T(x)] \dot{-} T(x) .
$$

By using Proposition 1 (ii) we yield

$$
T(x+\alpha(y-x)) \dot{-} T(x) \preceq^{m} \alpha T(y)-\alpha T(x) .
$$

Since $\preceq^{m}$ is compatible with the nonnegative scalar multiplication, we obtain

$$
\frac{T(x+\alpha(y-x)) \dot{-} T(x)}{\alpha} \preceq^{m} \frac{\alpha(T(y)-T(x))}{\alpha}=T(y)-T(x) .
$$

By taking Painlevé-Kuratowski upper limit for $\alpha \rightarrow 0^{+}$, we attain $T^{M}(x ; y-x) \preceq^{m}$ $T(y)-T(x)$ for all $x, y \in S$.

Remark 1 . Let $S \subseteq \mathbb{R}^{m}$ be a convex set and $T: \mathbb{R}^{m} \rightrightarrows \mathbb{R}^{n}$ be compact, convex valued and $M$-directionally differentiable set-valued mapping on $S$. If $T^{M}(x ; y-x) \preceq^{m}$ $T(y)-T(x)$ for all $x, y \in S, T$ may not be an $m$-convex set-valued mapping. For example, set-valued mapping $T: \mathbb{R} \rightrightarrows \mathbb{R}^{2}$ is defined as $T(x)=\mathbb{B}\left(\left(x, x^{2}\right),|1-x|\right)$ for all $x \in \mathbb{R}$, where $\mathbb{B}(x, r)$ denotes the open ball centered $x \in \mathbb{R}$ with radius $r$. Although $T$ is not $m$-convex set-valued mapping, the inequality condition $T^{M}(x ; y-x) \preceq^{m}$ $T(y)-T(x)$ is satisfied for all $x, y \in S$. 
Definition 8. Let $S \subseteq \mathbb{R}^{m}$ be a convex, $T: \mathbb{R}^{m} \rightrightarrows \mathbb{R}^{n}$ be an $M$-directionally differentiable. Then, $T$ is called

(i) $m$-pseudoconvex iff for all $x, y \in S$

$$
F^{M}(x ; y-x) \swarrow^{m} 0 \Longrightarrow F(y) \swarrow^{m} F(x),
$$

(ii) strongly $m$-pseudoconvex iff for all $x, y \in S$

$$
0 \preceq^{m} F^{M}(x ; y-x) \Longrightarrow F(x) \preceq^{m} F(y),
$$

(iii) weakly $m$-pseudoconvex iff for all $x, y \in S$

$$
F^{M}(x ; y-x) \nprec^{m} 0 \Longrightarrow F(y) \nprec^{m} F(x) .
$$

\section{SET VARIATIONAL INEQUALITY PROBLEMS AND OPTIMALITY CRITERIA FOR SET OPTIMIZATION}

Variational inequality problems and some optimality conditions for $\left(m-S V O_{P}\right)$ are introduced in this section.

Definition 9. Let $S \subseteq \mathbb{R}^{n}$ be a convex set and $x \in S$ be an arbitrary element. Then, the set-valued mapping $T: \mathbb{R}^{n} \rightrightarrows \mathbb{R}^{p}$ is called

(i) $m$-upper sign continuous if for all $y \in S$ and all $\alpha \in(0,1)$

$$
T(x+\alpha(y-x)) \swarrow^{m} 0 \Rightarrow T(x) \swarrow^{m} 0,
$$

(ii) strongly $m$-upper sign continuous if for all $y \in S$ and all $\alpha \in(0,1)$

$$
0 \preceq^{m} T(x+\alpha(y-x)) \Rightarrow 0 \preceq^{m} T(x),
$$

(iii) weakly $m$-upper sign continuous if for all $y \in S$ and all $\alpha \in(0,1)$

$$
T(x+\alpha(y-x)) \nprec^{m} 0 \Rightarrow T(x) \nprec^{m} 0 .
$$

The $m$-variational inequality problem (shortly $\left(m-V I_{P}\right)$ ): Find $x \in S$ such that

$$
T^{M}(x ; y-x) \swarrow^{m} 0, \quad \forall y \in S .
$$

The inverse $m$-variational inequality problem (shortly $\left(m-I V I_{P}\right)$ ): Find $x \in S$ such that

$$
0 \swarrow^{m} T^{M}(x ; y-x), \quad \forall y \in S .
$$

The strongly $m$-variational inequality problem (shortly $\left(m-S V I_{P}\right)$ ): Find $x \in S$ such that

$$
0 \preceq^{m} T^{M}(x ; y-x), \quad \forall y \in S .
$$

The inverse strongly $m$-variational inequality problem (shortly $\left(m-I S V I_{P}\right)$ ): Find $x \in S$ such that 


$$
T^{M}(x ; y-x) \preceq^{m} 0, \quad \forall y \in S .
$$

Similarly, the weakly $m$-variational inequality problem (shortly $\left.\left(m-W V I_{P}\right)\right)$ : Find $x \in S$ such that

$$
T^{M}(x ; y-x) \nprec^{m} 0, \quad \forall y \in S .
$$

The inverse weakly $m$-variational inequality problem (shortly $\left.\left(m-I W V I_{P}\right)\right)$ : Find $x \in S$ such that

$$
0 \nprec^{m} T^{M}(x ; y-x), \quad \forall y \in S .
$$

We denote by $\operatorname{sol}\left(m-S V I_{P}\right), \operatorname{sol}\left(m-V I_{P}\right)$ and $\operatorname{sol}\left(m-W V I_{P}\right)$ the set of all solutions of $\left(m-S V I_{P}\right),\left(m-V I_{P}\right)$ and $\left(m-W V I_{P}\right)$, respectively. It is obvious that $\operatorname{sol}\left(m-S V I_{P}\right) \subseteq \operatorname{sol}\left(m-V I_{P}\right) \subseteq \operatorname{sol}\left(m-W V I_{P}\right)$. Similarly, there is the same relationship in the inverse version of the variational inequality problems. The converse implications may not be generally true.

Minty $m$-variational inequality problem $\left(m-M V I_{P}\right)$ : Find $x \in S$ so that

$$
T^{M}(y ; y-x) \swarrow^{m} 0, \quad \forall y \in S,
$$

Minty strongly $m$-variational inequality problem $\left(m-M S V I_{P}\right)$ : Find $x \in S$ so that

$$
0 \preceq^{m} T^{M}(y ; y-x), \quad \forall y \in S,
$$

Minty weakly $m$-variational inequality problem $\left(m-M W V I_{P}\right)$ : Find $x \in S$ so that

$$
T^{M}(y ; y-x) \nprec^{m} 0, \quad \forall y \in S .
$$

We denote by $\operatorname{sol}\left(m-M S V I_{P}\right), \operatorname{sol}\left(m-M V I_{P}\right)$ and $\operatorname{sol}\left(m-M W V I_{P}\right)$ the set of all solutions of $\left(m-M S V I_{P}\right),\left(m-M V I_{P}\right)$ and $\left(m-M W V I_{P}\right)$, respectively. It is obvious that $\operatorname{sol}\left(m-M S V I_{P}\right) \subseteq \operatorname{sol}\left(m-M V I_{P}\right) \subseteq \operatorname{sol}\left(m-M W V I_{P}\right)$, but the converse inclusion may not be generally true. Similarly, there is the same relationship in inverse version of variational inequality problems.

Proposition 6. Let $S \subseteq \mathbb{R}^{n}$ be a nonempty convex set and set-valued mapping $T: \mathbb{R}^{n} \rightrightarrows \mathbb{R}^{p}$ be M-directionally differentiable on $S$. Then

(i) if $T^{M}$ is m-upper sign continuous, then $\operatorname{sol}\left(m-M V I_{P}\right) \subseteq \operatorname{sol}\left(m-V I_{P}\right)$,

(ii) if $T^{M}$ is strongly m-upper sign continuous, then $\operatorname{sol}\left(m-M S V I_{P}\right) \subseteq \operatorname{sol}(m-$ $\left.S V I_{P}\right)$,

(iii) if $T^{M}$ is weakly m-upper sign continuous, then $\operatorname{sol}\left(m-M W V I_{P}\right) \subseteq \operatorname{sol}(m-$ $\left.W V I_{P}\right)$.

Proof. (i) Let $x^{\prime} \in S$ is a solution of $\left(m-M V I_{P}\right)$. Then, $T^{M}\left(y ; y-x^{\prime}\right) \swarrow^{m} 0$ for all $y \in S$. Since $S$ is a convex set, $x^{\prime}+\lambda\left(y-x^{\prime}\right) \in S$ for all $\lambda \in(0,1)$. So, we have

$$
T^{M}\left(x^{\prime}+\lambda\left(y-x^{\prime}\right) ; x^{\prime}+\lambda\left(y-x^{\prime}\right)-x^{\prime}\right) \swarrow^{m} 0,
$$


equivalently from positively homogenously of $M$-directionally differentiable

$$
\lambda T^{M}\left(x^{\prime}+\lambda\left(y-x^{\prime}\right) ; y-x^{\prime}\right) \npreceq^{m} 0 .
$$

By Proposition 2 (iv), we yield $T^{M}\left(x^{\prime}+\lambda\left(y-x^{\prime}\right) ; y-x^{\prime}\right) \swarrow^{m} 0 . T^{M}\left(x^{\prime} ; y-\right.$ $\left.x^{\prime}\right) \swarrow^{m} 0$ yields by using $m$-upper sign continuity of $T^{M}$. Therefore, $x^{\prime} \in$ $\operatorname{sol}\left(m-V I_{P}\right)$.

(ii-iii) These can be proven similar to (i).

Theorem 2. Let $S \subseteq \mathbb{R}^{m}$ be a convex set and set-valued map $T: \mathbb{R}^{m} \rightrightarrows \mathbb{R}^{n}$ be compact, convex valued and $M$-directionally differentiable on $S$. Then, $x_{0}$ is a maximal solution of $\left(m-S V O_{P}\right)$ if and only if it is also a solution of $\left(m-I V I_{P}\right)$.

Proof. Let $x_{0}$ be a maximal solution of $\left(m-S V O_{P}\right)$. We have

$$
T\left(x_{0}\right) \swarrow^{m} T(y)
$$

for all $y \in S \backslash\left\{x_{0}\right\}$ such that $T(y) \neq T\left(x_{0}\right)$. Then, we can obtain easily that

$$
0 \swarrow^{m} T(y) \dot{-} T\left(x_{0}\right) \text {. }
$$

Because $S$ is convex set, we can write $\alpha y+(1-\alpha) x_{0}$ instead of $y$ in (4.2) for $\alpha \in$ $(0,1)$. Hence,

$$
0 \swarrow^{m} T\left(x_{0}+\alpha\left(y-x_{0}\right)\right) \dot{-} T\left(x_{0}\right) .
$$

From Proposition 2 (iv), we have

$$
0 \swarrow^{m} \frac{T\left(x_{0}+\alpha\left(y-x_{0}\right)\right) \dot{-} T\left(x_{0}\right)}{\alpha} .
$$

Since $T$ is $M$-directionally differentiable and $T\left(x_{0}+\alpha\left(y-x_{0}\right)\right) \dot{-} T\left(x_{0}\right)$ is compact, by taking Painlevé-Kuratowski upper limit $\alpha \rightarrow 0^{+}$in (4.3) we yield $0 \swarrow^{m} T^{M}\left(x_{0} ; y-x_{0}\right)$. Therefore, $x_{0}$ is a solution of $\left(m-I V I_{P}\right)$.

For the inverse statement, let $x_{0}$ be a solution of $0 \swarrow^{m} T^{M}(x ; y-x)$ for all $y \in S$. Assume that $x_{0}$ is not a solution of $\left(m-S V O_{P}\right)$. There exists an $x^{\prime} \in S$ such that

$$
T\left(x_{0}\right) \preceq^{m} T\left(x^{\prime}\right) .
$$

Since $S$ is convex set and $x_{0}, x^{\prime} \in S$, there exists a $y \in S$ and $\alpha \in[0,1]$ such that $x^{\prime}=$ $\alpha y+(1-\alpha) x_{0}$. From (4.4) and Proposition 2 (iii), $0 \preceq^{m} T\left(x_{0}+\alpha\left(y-x_{0}\right)\right) \dot{-} T\left(x_{0}\right)$ yield. By multiplying both sides with $\frac{1}{\alpha}$ and by taking Painlevé-Kuratowski upper limit $\alpha \rightarrow 0^{+}$, we obtain $0 \preceq^{m} T^{M}\left(x_{0} ; y-x_{0}\right)$. This contradicts the assumption. Hence, $x_{0}$ is a maximal solution of $\left(m-S V O_{P}\right)$.

Theorem 3. Let $S \subseteq \mathbb{R}^{m}$ be convex set and set-valued map $T: \mathbb{R}^{m} \rightrightarrows \mathbb{R}^{n}$ be compact, convex valued and $M$-directionally differentiable on $S$. Then, $x_{0}$ is a minimal solution of $\left(m-S V O_{P}\right)$ if and only if it is a solution of $\left(m-V I_{P}\right)$.

Proof. It can be obtained similar to the proof of previous theorem. 
Theorem 4. Let $S \subseteq \mathbb{R}^{m}$ be convex set and set-valued map $T: \mathbb{R}^{m} \rightrightarrows \mathbb{R}^{n}$ be compact, convex valued and $M$-directionally differentiable on $S$. Then,

(i) $x_{0}$ is a strongly minimal (resp. strongly maximal) solution of $\left(m-S V O_{P}\right)$ if and only if it is also a solution of $\left(m-S V I_{P}\right)\left(\right.$ resp. $\left.\left(m-I S V I_{P}\right)\right)$,

(ii) $x_{0}$ is a weakly minimal (resp. weakly maximal) solution of $\left(m-S V O_{P}\right)$ if and only if it is also a solution of $\left(m-W V I_{P}\right)$ (resp. $\left.\left(m-I W V I_{P}\right)\right)$.

Proposition 7. Let $S \subseteq \mathbb{R}^{m}$ be convex set and set-valued map $T: \mathbb{R}^{m} \rightrightarrows \mathbb{R}^{n}$ be compact, convex valued and $M$-directionally differentiable on $S$. Then,

(i) if $\tilde{x}$ is a strongly minimal (resp. strongly maximal) solution of $\left(m-S V O_{P}\right)$, then $\tilde{x}$ is also not only a solution of $\left(m-V I_{P}\right)\left(\right.$ resp. $\left.\left(m-I V I_{P}\right)\right)$ but also a solution of $\left(m-W V I_{P}\right)$ (resp. $\left.\left(m-I W V I_{P}\right)\right)$,

(ii) if $\tilde{x}$ is a minimal (resp. maximal) solution of $\left(m-S V O_{P}\right)$, then $\tilde{x}$ is also a solution of $\left(m-W V I_{P}\right)$ (resp. $\left.\left(m-I W V I_{P}\right)\right)$.

Theorem 5. Let $S \subseteq \mathbb{R}^{m}$ be convex set and $T: \mathbb{R}^{m} \rightrightarrows \mathbb{R}^{n}$ be $M$-directionally differentiable set-valued map on $S \subseteq$. The following assertions are ture:

(i) if $T$ is m-pseudoconvex, then every solution of $\left(m-V I_{P}\right)$ is a minimal solution of $\left(m-S V O_{P}\right)$,

(i) if $T$ is $m$-pseudoconvex, then every solution of $\left(m-M V I_{P}\right)$ is a maximal solution of $\left(m-S V O_{P}\right)$,

(i) if $T$ is weakly m-pseudoconvex, then every solution of $\left(m-W V I_{P}\right)$ is a weak minimal solution of $\left(m-S V O_{P}\right)$,

(i) if $T$ is weakly m-pseudoconvex, then every solution of $\left(m-M W V I_{P}\right)$ is a weak maximal solution of $\left(m-S V O_{P}\right)$,

(ii) if $T$ is strongly m-pseudoconvex, every solution of $\left(m-S V I_{P}\right)$ is also a strongly minimal solution of $\left(m-S V O_{P}\right)$,

(ii) if $T$ is strongly m-pseudoconvex, every solution of $\left(m-M S V I_{P}\right)$ is also a strongly maximal solution of $\left(m-S V O_{P}\right)$.

Proof. The proof can be proved easily by using the definitions.

\section{REFERENCES}

[1] F. Abdolrazaghi and A. Razani, "On the weak solutions of an overdetermined system of nonlinear fractional partial integro-differential equations." Miskolc Mathematical Notes, vol. 20, no. 1, pp. 3-16, 2019, doi: 10.18514/MMN.2019.2755.

[2] Q. H. Ansari, E. Köbis, and J.-C. Yao, Vector variational inequalities and vector optimization: theory and applications. Berlin, Heidelberg: Springer-Verlag, 2018. doi: 10.1007/978-3-31963049-6.

[3] Q. H. Ansari and G. M. Lee, "Nonsmooth vector optimization problems and minty vector variational inequalities." J. Optim. Theory. Appl., vol. 145, no. 1, p. 1-16, 2010, doi: 10.1007/s10957009-9638-9. 
[4] G. Y. Chen, X. Huang, and X. Yang, Vector optimization: set-valued and variational analysis. Berlin, Heidelberg: Springer Science and Business Media, 2006, vol. 541, doi: 10.1007/3-54028445-1.

[5] Y. Q. Chen, "On the semi-monotone operator theory and applications." J. Math. Anal. Appl., vol. 231, p. 177-192, 1999, doi: 10.1006/jmaa.1998.6245.

[6] G. P. Crespi, I. Ginchev, and M. Rocca, "Existence of solutions and star-shapedness in minty variational inequalities.” J. Glob. Optim., vol. 32, no. 4, p. 485-494, 2005, doi: 10.1007/s10898003-2685-0.

[7] G. P. Crespi and I. G. M. Rocca, "Minty variational inequalities, increase along rays property and optimization." J. Optim. Theory Appl., vol. 123, no. 3, p. 479-496, 2004, doi: 10.1007/s10957004-5719-y.

[8] F. Giannessi, Theorems of alternative, quadratic programs and complementarity problems, in Variational Inequalities and Complementarity Problems., R. W. Cottle, F. Giannessi, and J. L. Lions, Eds. New York: John Wiley and Sons, 1980. doi: 10.1007/3-540-28445-1.

[9] E. Hernández and L. Rodríguez-Marín, "Weak and strongly subgradients of set-valued maps." $J$. Optim. Theory. Appl., vol. 149, no. 2, pp. 352-365, 2011, doi: 10.1007/s10957-010-9787-x.

[10] J. Jahn, "Vectorization in set optimization." J. Optimiz. Theory. App., vol. 167, pp. 783-795, 2013, doi: 10.1007/s10957-013-0363-z.

[11] E. Karaman, I. Atasever Güvenç, and M. Soyertem, "Optimality conditions in set-valued optimization problems with respect to a partial order relation by using subdifferentials." Optimization, 2020, doi: 10.1080/02331934.2020.1728270.

[12] E. Karaman, I. Atasever Güvenç, M. Soyertem, D. Tozkan, M. Küçük, and Y. Küçük, "A vectorization for nonconvex set-valued optimization.” Turk. J. Math., vol. 42, pp. 1815-1832, 2018, doi: 10.3906/mat-1707-75.

[13] E. Karaman, M. Soyertem, and I. Atasever Güvenç, " "Optimality conditions in set-valued optimization problem with respect to a partial order relation via directional derivative." Taiwan. J. Math., 2020, doi: 10.11650/tjm/190604.

[14] E. Karaman, M. Soyertem, I. Atasever Güvenç, D. Tozkan, M. Küçük, and Y. Küçük, "Partial order relations on family of sets and scalarizations for set optimization." Positivity, vol. 22, no. 3, pp. 783-802, 2018, doi: 10.1007/s11117-017-0544-3.

[15] E. Karaman, "Gömme fonksiyonu kullanılarak küme optimizasyonuna göre verilen küme değerli optimizasyon problemlerinin optimallik koşulları.” Süleyman Demirel Üniversitesi Fen Edebiyat Fakültesi Fen Dergisi, vol. 14, pp. 105-111, 2019, doi: 10.29233/sdufeffd.481206.

[16] D. Kuroiwa, "On set-valued optimization." Nonlinear Anal-Theor., vol. 47, no. 2, pp. 1395-1400, 2001, doi: 10.1016/S0362-546X(01)00274-7.

[17] D. Pallaschke and R. Urbański, Pairs of compact convex sets. Dordrecht: Kluwer academic publishers, 2002. doi: 10.1007/978-94-015-9920-7.

[18] M. A. Ragusa, "Embeddings for morrey-lorentz spaces." J. Optimiz. Theory. App., vol. 154, no. 2, p. 491-499, 2012, doi: 10.1007/s10957-012-0012-y.

[19] M. A. Ragusa and A. Tachikawa, "Regularity of minimizers of some variational integrals with discontinuity." Zeitschrift für Analysis und ihre Anwendungen, vol. 27, no. 4, p. 469-482, 2008, doi: 10.4171/ZAA/1366.

[20] R. Schneider, Convex Bodies: The Brunn-Minkowski theory, second expanded edition, Encyclopedia of Mathematics and its Applications. Cambridge: Cambridge University Press, 2014. doi: 10.1017/CBO9781139003858.

[21] H. Yin, C. X. Xu, and Z. X. Zhang, "The F-complementarity problems and its equivalence with the least element problem.” Acta Math. Sin., vol. 44, no. 4, p. 679-686, 2001. 
Author's address

\section{E. Karaman}

Karabük University, Faculty of Science, Department of Mathematics, 78050 Karabük, Turkey

E-mail address: e.karaman42@gmail.com 